TRANSACTIONS OF THE

AMERICAN MATHEMATICAL SOCIETY

Volume 363, Number 5, May 2011, Pages 2641-2661

S 0002-9947(2010)05206-7

Article electronically published on December 22, 2010

\title{
LEAST ACTION PRINCIPLE AND THE INCOMPRESSIBLE EULER EQUATIONS WITH VARIABLE DENSITY
}

\author{
MILTON C. LOPES FILHO, HELENA J. NUSSENZVEIG LOPES, \\ AND JULIANA C. PRECIOSO
}

\begin{abstract}
In this article we study a variational formulation, proposed by V. I. Arnold and by Y. Brenier, for the incompressible Euler equations with variable density. We consider the problem of minimizing an action functional, the integral in time of the kinetic energy, among fluid motions considered as trajectories in the group of volume-preserving diffeomorphisms beginning at the identity and ending at some fixed diffeomorphism at a given time. We show that a relaxed version of this variational problem always has a solution, and we derive an Euler-Lagrange system for the relaxed minimization problem which we call the relaxed Euler equations. Finally, we prove consistency between the relaxed Euler equations and the classical Euler system, showing that weak solutions of the relaxed Euler equations with the appropriate geometric structure give rise to classical Euler solutions and that classical solutions of the Euler system induce weak solutions of the relaxed Euler equations. The first consistency result is new even in the constant density case. The remainder of our analysis is an extension of the work of Y. Brenier (1999) to the variable density case.
\end{abstract}

\section{INTRODUCTION}

The motion of an incompressible inviscid fluid can be described by the Euler equations. In 1966, V. I. Arnold observed that incompressible inviscid flows can be interpreted as geodesics on the group of volume-preserving maps endowed with the left-invariant Riemannian structure inherited from the Lie algebra of divergence-free vector fields with the $L^{2}$ metric.

To be more precise, let $D \subset \mathbb{R}^{N}$ be a compact domain and consider

$$
G(D)=\left\{h \in C^{1}(D ; D) \mid \operatorname{det} D_{x} h=1 ; h \text { a diffeomorphism }\right\},
$$

the set of volume-preserving $C^{1}$-diffeomorphisms of $D$, endowed with the leftinvariant Riemannian structure from 2. Consider a solution $u=u(t, x)$ of the incompressible Euler equations in $D$. If $(t, x) \mapsto g_{u}(t, x)$ denotes the flow induced by $u$, then each trajectory $t \mapsto g_{u}(t, x)$ is a geodesic on $G(D)$.

Received by the editors January 27, 2009 and, in revised form, August 24, 2009 and September 2, 2009.

2010 Mathematics Subject Classification. Primary 35Q31; Secondary 49 J45.

The first author's research was partially supported by CNPq Grant \#303301/2007-4.

The second author's research was partially supported by CNPq Grant \#302214/2004-6.

The third author's research was part of the Ph.D. thesis of J. C. Precioso, partially supported by FAPESP Grant \#2001/06984-5. 
Let $g=g(t)$ be a path on $G(D)$. The action is defined as

$$
A=A(g) \equiv \frac{1}{2} \int_{0}^{T}\left\|\partial_{t} g\right\|_{L^{2}}^{2} d t
$$

for some fixed $T>0$.

By Arnold's construction, the existence of minimal geodesics in $G(D)$ is equivalent to the existence of minimizers of the action. The existence and uniqueness of minimal geodesics was first established in 1970, by D. G. Ebin and J. Marsden, see [9], for target diffeomorphisms which are both sufficiently smooth and sufficiently close to the identity. However, the existence of minimizing geodesics is not true in the large. In [13, Shnirelman showed that there are "targets" $h \in G(D)$, with $D=[0,1]^{3}$, for which there is no minimal geodesic connecting $h$ to the identity map.

We will focus on the case $D=[0,1]^{N}$. In [7, Yann Brenier introduced a weak formulation of the problem of existence of minimal geodesics in the case of homogeneous (constant density) fluids, for which he showed existence of a solution. This formulation was inspired by Monge-Kantorovich theory and it consists of reinterpreting the geodesic as a measure $c$ on $[0, T] \times D \times D$, with $(x, a) \in D \times D$ representing the position $x$ and the Lagrangian marker $a$, while relaxing the minimization problem. For a discussion of the connection between this problem and optimal mass transportation, see [16]. The solution to the relaxed problem is a pair of measures $(c, m)$, where $m$ is a vector-valued measure representing a weak version of the Euler flow $u$. The Euler-Lagrange equations associated to the relaxed problem are called relaxed Euler equations. Recently, Ambrosio and Figalli showed an improved regularity result for the pressure associated with Brenier's optimal solutions; see 1 .

In this paper, we extend Brenier's work to the case of incompressible fluids with variable density, deriving a nonhomogeneous version of the relaxed Euler equations. Our argument follows closely that of Brenier. We will present the main ideas in Brenier's result, and we give detailed proofs only where a nontrivial adaptation is needed.

Additionally, we prove consistency of the relaxed nonhomogeneous Euler equations with the classical nonhomogeneous incompressible Euler equations, which amounts to two results. In the first, we prove that weak solutions of the relaxed Euler equations with the appropriate geometric structure and regularity give rise to classical solutions of the Euler equations. In the second result, we prove that classical solutions of the Euler equations induce solutions of the relaxed Euler equations, and that, in a sufficiently small time interval, this induced solution is the unique minimizer of the relaxed problem. The first consistency result is new, even in the context of homogeneous flows, whereas the second result is a straightforward generalization of Theorem 2.4 in [7.

Variable density incompressible Euler equations are a physically interesting model for which very little is known beyond local existence of smooth solutions for the Cauchy problem; see 4, 5, 8. In two space dimensions, this situation contrasts sharply with the homogeneous case, for which there is a well-developed theory of weak solutions; see [11 and the references therein. This is one motivation behind the present work. We note that the variational formulation provides an avenue for the study of weak solutions of the nonhomogeneous Euler system, and the existence of optimal solutions implies the existence of a large class of generalized solutions, 
though, unfortunately, not for the Cauchy problem. The consistency results provide the framework in which to understand the relaxed Euler system as a generalized formulation of the variable density Euler system.

The remainder of this article is divided into four sections. In Section 2 we write the Lagrangian formulation of the Euler equations and introduce basic notation. In Section 3 we formulate the relaxed problem. In Section 4 we discuss the existence of optimal solutions to the relaxed problem and in Section 5 we state and prove the consistency results.

\section{The Least aCtion PRinciple and the Euler EQuations}

In this section we rewrite the nonhomogeneous incompressible Euler equations in Lagrangian form and we interpret the Lagrangian flow as a minimizer of an appropriate action.

Fix $D=[0,1]^{N}$, the unit cube in $\mathbb{R}^{N}$, let $T>0$ and set $Q=[0, T] \times D$. The motion of a nonhomogeneous incompressible inviscid fluid in $D$ can be described by the incompressible Euler equations:

$$
\left\{\begin{array}{l}
\rho\left[\partial_{t} u+(u \cdot \nabla) u\right]+\nabla p=0, \\
\partial_{t} \rho+u \cdot \nabla \rho=0, \\
\nabla \cdot u=0, \\
u(t, \cdot) \cdot \hat{n}_{\partial D}=0 .
\end{array}\right.
$$

Above, $\rho=\rho(t, x)$ denotes the density of the fluid, $u=u(t, x)$ is the velocity field and $p=p(t, x)$ is the scalar pressure. The vector $\hat{n}$ is the exterior unit normal to the cube. For smooth solutions, the boundary condition should be understood as tangency of the solution to the boundary or, in other words, if a point $P$ at the boundary lies at the intersection of several faces, the velocity $u(P)$ should be orthogonal to the normal vector of all the intersecting faces. We emphasize that we consider flows in an $N$-dimensional cube with nonpenetration boundary conditions; not periodic flows.

Let

$$
V \equiv\left\{u: Q \rightarrow \mathbb{R}^{N} \mid u \in C^{0}(Q) \cap C([0, T] ; \operatorname{Lip}(D)), \operatorname{div} u=0, u(t, \cdot) \cdot \widehat{n}_{\left.\right|_{\partial D}}=0\right\} .
$$

For each $u \in V$ and $x \in D$ let $g_{u}=g_{u}(t, x)$ denote the flow induced by $u$ with initial position $x$. Since $\operatorname{div} u=0$ it follows that $g_{u}(t, \cdot) \in G(D)$.

The incompressible Euler equations (2.1) can be rewritten in Lagrangian form as

$$
\left\{\begin{array}{l}
\rho_{0}(x) \partial_{t t}^{2} g(t, x)+\nabla p(t, g(t, x))=0 \\
\rho(t, g(t, x))=\rho(t=0, x)=\rho_{0}(x) \\
\operatorname{det} D_{x} g(t, x)=1
\end{array}\right.
$$

In 2], Arnold considered the homogeneous case $\rho_{0} \equiv 1$ and observed that, if $g=g(t, x)$ is the Lagrangian flow associated with a solution of the (homogeneous) Euler equations, then $g$ is a geodesic in the group $G(D)$.

In the homogeneous context, a minimal geodesic $g$ between the identity and $h \in G(D)$ also minimizes the action:

$$
A(g)=\frac{1}{2} \int_{0}^{T} \int_{D}\left|\frac{\partial g}{\partial t}(t, x)\right|^{2} d x d t
$$


among all smooth paths $t \in[0, T] \mapsto g(t, \cdot) \in G(D)$ such that

$$
g(0)=i d \text { and } g(T)=h .
$$

As observed in [6], there is also a least action principle for solutions of (2.2), where the action is now given by

$$
A_{\rho_{0}}=A_{\rho_{0}}(g) \equiv \frac{1}{2} \int_{0}^{T} \int_{D} \rho_{0}(x)\left|\partial_{t} g(t, x)\right|^{2} d x d t .
$$

If $g$ is a smooth solution of (2.2), then $g$ is a minimizer of the action $A_{\rho_{0}}$; see [6].

Given $g=g(t)$ a smooth path on $G(D)$ set $\rho=\rho(t, y) \equiv \rho_{0}\left(t,\left(g(t, \cdot)^{-1}(y)\right)\right.$ and define the vector field $u=u(t, x) \equiv \partial_{t} g\left(t,(g(t, \cdot))^{-1}(x)\right)$, so that $\partial_{t} g(t, y)=$ $u(t, g(t, y))$. Then, switching back to Eulerian coordinates, the problem of minimizing the action $A_{\rho_{0}}$ can be reformulated in terms of the functional

$$
K=K[u]=\frac{1}{2} \int_{Q}|u(t, y)|^{2} \rho(t, y) d y d t
$$

where $u \in V$ is such that $g_{u}(T, \cdot)=h(\cdot)$ and where $\rho\left(t, g_{u}(t, x)\right)=\rho_{0}(x)$.

\section{The RELAXED PRoBlem}

A large part of the difficulty involved in solving the minimization problem for the action (or for the functional in (2.4) ) is the nonlinear constraint that $g \in G(D)$. As is standard in convex analysis we will consider a relaxed problem where this nonlinear constraint is imbedded into a larger, linear, admissible set.

We will assume throughout the remainder of this work that

$$
\rho_{0} \in C^{0}(D), \quad \rho_{0}>0 .
$$

Set $Q^{\prime}=Q \times D$, where $Q=[0, T] \times D$. For each $\mu \in \mathcal{B M}\left(Q^{\prime}\right)$, we define a measure $\mu_{\rho_{0}}$ through the pairing

$$
\left\langle\mu_{\rho_{0}}, f\right\rangle \equiv\left\langle\mu, \rho_{0} f\right\rangle
$$

for any test function $f \in C^{0}\left(Q^{\prime}\right)$. We will be working with functions that depend both on Eulerian variables $(x)$ and on Lagrangian variables $(a)$ simultaneously; for the sake of clarity, we will denote by $D_{x}$ the unit cube in the $x$ variables and by $D_{a}$ the same cube in the $a$ variables.

For each $u \in V$ let $g=g(t, a)=g_{u}(t, a)$ be the associated flow. Let $h=h(a)=$ $g(T, a)$ and note that $h \in G(D)$. Consider

$$
\Sigma=\left\{(t, g(t, a), a) \mid t \in[0, T], a \in D_{a}\right\} \subset Q^{\prime} \subset \mathbb{R}^{2 N+1} .
$$

Let $\delta_{(t, g(t, a), a)} \in \mathcal{B M}\left(Q^{\prime}\right)$ denote the measure such that

$$
\left\langle\delta_{(t, g(t, a), a)}, f\right\rangle=\int_{0}^{T} \int_{D_{a}} f(t, g(t, a), a) d a d t
$$

for any $f \in C^{0}\left(Q^{\prime}\right)$.

We introduce $(c, m) \in \mathcal{B M}\left(Q^{\prime}\right) \times\left(\mathcal{B M}\left(Q^{\prime}\right)\right)^{N}$ as

$$
(c, m) \equiv\left(\delta_{(t, g(t, a), a)}, u(t, x) \delta_{(t, g(t, a), a)}\right) .
$$


More precisely, for any $f \in C^{0}\left(Q^{\prime}\right)$, it follows that

$$
\begin{gathered}
\langle m, f\rangle=\int_{Q^{\prime}} f(t, x, a) d m(t, x, a) \\
=\int_{Q} f(t, g(t, a), a) u(t, g(t, a)) d a d t=\int_{Q} f(t, g(t, a), a) \partial_{t} g(t, a) d a d t .
\end{gathered}
$$

Consider the pair $\left(c_{\rho_{0}}, m_{\rho_{0}}\right)$ associated with $(c, m)$ through (3.2). Then, for any $f \in C^{0}\left(Q^{\prime}\right)$ such that $\partial_{t} f$ and $\nabla_{x} f$ are continuous in $Q^{\prime}$, an easy calculation shows that:

$$
\left\langle c_{\rho_{0}}, \partial_{t} f\right\rangle+\left\langle m_{\rho_{0}}, \nabla_{x} f\right\rangle=\int_{D_{a}} \rho_{0}(a)[f(T, h(a), a)-f(0, a, a)] d a .
$$

In particular, the continuity equation holds:

$$
\partial_{t} c_{\rho_{0}}+\nabla_{x} \cdot m_{\rho_{0}}=0
$$

in the distributional sense of $\mathcal{D}^{\prime}\left(Q^{\prime}\right)$.

Next, note that, if $\bar{f}=\bar{f}(t, x) \in C^{0}(Q)$, then, since $1 \in C^{0}\left(D_{a}\right)$, it follows that $f=\bar{f} \cdot 1 \in C^{0}\left(Q^{\prime}\right)$. Hence we find:

$$
\left\langle\frac{c_{\rho_{0}}}{\rho_{0}}, f\right\rangle=\int_{Q} \bar{f}(t, g(t, a)) d t d a=\int_{Q} \bar{f}(t, x) d t d x,
$$

which we can interpret as

$$
\int_{D_{a}} \frac{c_{\rho_{0}}(t, x, a)}{\rho_{0}(a)} d a=1 .
$$

Analogously to what was done in [7, we rewrite $K[u]$ (see (2.4)), in terms of the measures $\left(c_{\rho_{0}}, m_{\rho_{0}}\right)$ as follows:

$$
K[u]=K\left[c_{\rho_{0}}, m_{\rho_{0}}\right]=\sup _{X}\left\{\left\langle c_{\rho_{0}}, F\right\rangle+\left\langle m_{\rho_{0}}, \Phi\right\rangle\right\}
$$

where

$$
X=\left\{(F, \Phi) \in C^{0}\left(Q^{\prime}\right) \times\left.\left(C^{0}\left(Q^{\prime}\right)\right)^{N}\left|F(t, x, a)+\frac{1}{2}\right| \Phi(t, x, a)\right|^{2} \leq 0\right\} .
$$

We have described, for each $u \in V$, the construction of a pair $\left(c_{\rho_{0}}, m_{\rho_{0}}\right)$ of measures such that (3.4) and (3.6) hold and (3.8) follows. This construction serves as motivation for the definition of the relaxed problem, which we make precise below.

First define

$$
\begin{aligned}
& S(D)=\{h: D \rightarrow D \mid h \text { is measurable and } \\
& \left.\qquad \int_{D} f(h(x)) d x=\int_{D} f(x) d x, \quad \forall f \in C^{0}(D)\right\} .
\end{aligned}
$$

Let $h \in S(D)$. We introduce an admissible set $\mathcal{A}$ by:

$$
\begin{gathered}
\mathcal{A}=\left\{\left(c_{\rho_{0}}, m_{\rho_{0}}\right) \in \mathcal{B M}\left(Q^{\prime}\right) \times\left(\mathcal{B M}\left(Q^{\prime}\right)\right)^{N} \mid K\left[c_{\rho_{0}}, m_{\rho_{0}}\right]<+\infty\right. \text { and } \\
\left.\left(c_{\rho_{0}}, m_{\rho_{0}}\right) \text { satisfy (3.4) for all } f \in C^{1}\left(Q^{\prime}\right) \text { and (3.6) for all } \bar{f} \in C^{0}(Q)\right\} .
\end{gathered}
$$


Relaxed Problem: Find a pair $\left(\bar{c}_{\rho_{0}}, \bar{m}_{\rho_{0}}\right) \in \mathcal{A}$ such that

$$
K\left[\bar{c}_{\rho_{0}}, \bar{m}_{\rho_{0}}\right]=\min _{\left(c_{\rho_{0}}, m_{\rho_{0}}\right) \in \mathcal{A}} K\left[c_{\rho_{0}}, m_{\rho_{0}}\right] .
$$

Note that the admissible set $\mathcal{A}$ depends on the final state $h$.

Definition 3.1. A pair of measures $\left(\bar{c}_{\rho_{0}}, \bar{m}_{\rho_{0}}\right)$ is an optimal solution if it satisfies (3.9). We denote the minimum value as

$$
K\left[\bar{c}_{\rho_{0}}, \bar{m}_{\rho_{0}}\right]=I^{*}[h] .
$$

\section{Existence of optimal solutions}

In this section we discuss the extension to nonhomogeneous fluids of Brenier's theorem on existence of generalized geodesics, as stated below.

Theorem 4.1. Let $h \in S(D)$. Then we have:

(A) There exists at least one optimal solution $\left(\bar{c}_{\rho_{0}}, \bar{m}_{\rho_{0}}\right)$ of the relaxed problem (3.9).

(B) There exists $v \in\left(L^{2}\left(Q^{\prime}, d \bar{c}_{\rho_{0}}\right)\right)^{N}$ such that $\bar{m}_{\rho_{0}}=v \bar{c}_{\rho_{0}}$.

(C) The kinetic energy,

$$
\int_{D_{x} \times D_{a}} \frac{1}{2}|v(t, x, a)|^{2} \bar{c}_{\rho_{0}}(t, d x, d a),
$$

is time-independent and bounded.

Furthermore, there exists a unique measure $\nabla_{x} p=\nabla_{x} p(t, x),(t, x) \in \stackrel{\circ}{Q}$, depending only on $h$, such that

$$
\partial_{t} \bar{m}_{\rho_{0}}+\nabla_{x} \cdot\left(v \otimes \bar{m}_{\rho_{0}}\right)+\underline{c} \nabla_{x} p=0
$$

in the sense of distributions in the interior of $Q^{\prime}$, for all optimal solutions $\left(\bar{c}_{\rho_{0}}, \bar{m}_{\rho_{0}}\right)$, where $\underline{c}$ is a certain extension of $\frac{\bar{c}_{\rho_{0}}}{\rho_{0}}$ for which the product $\underline{c} \nabla_{x} p$ is well-defined.

Observation: The system comprised of (3.5), (3.7) and (4.2) will be referred to as the relaxed (nonhomogeneous) Euler equations.

In the remainder of this section we will discuss the main ideas in the proof of Theorem 4.1. There are just a couple of minor points where adaptation to the nonhomogeneous case is needed, which are expressed below in the proofs of Propositions 4.3 and 4.4. In addition, for completeness' sake, we present an outline of Brenier's complete argument.

Lemma 4.2. We have $K\left[c_{\rho_{0}}, m_{\rho_{0}}\right]<\infty$ if and only if $c_{\rho_{0}}$ is a nonnegative measure, $m_{\rho_{0}} \ll c_{\rho_{0}}$ and $m_{\rho_{0}}=v c_{\rho_{0}}$ with $v \in L^{2}\left(Q^{\prime}, d c_{\rho_{0}}\right)^{N}$.

This result is a straightforward adaptation of the analogous result in the homogeneous case, discussed in 7]. The proof follows easily from the definition of $K\left[c_{\rho_{0}}, m_{\rho_{0}}\right]$ and the Radon-Nikoým Theorem.

Proposition 4.3. Let $h \in S(D)$. There exists an optimal solution $\left(\bar{c}_{\rho_{0}}, \bar{m}_{\rho_{0}}\right)$ of (3.9); i.e., there exists a pair $\left(\bar{c}_{\rho_{0}}, \bar{m}_{\rho_{0}}\right) \in \mathcal{A}$ such that $I^{*}[h]=K\left[\bar{c}_{\rho_{0}}, \bar{m}_{\rho_{0}}\right]<+\infty$. 
Proof of Proposition 4.3. Let us fix an admissible solution $\left(c_{\rho_{0}}, m_{\rho_{0}}\right)$ and consider the Banach space $E=C\left(Q^{\prime}\right) \times\left(C\left(Q^{\prime}\right)\right)^{N}$. For $(F, \Phi) \in E$ we define two convex functions

$$
\alpha_{\rho_{0}}=\alpha_{\rho_{0}}(F, \Phi) \equiv \alpha\left(\rho_{0} F, \Phi\right) \text { and } \beta_{\rho_{0}}=\beta_{\rho_{0}}(F, \Phi) \equiv \beta(F, \Phi),
$$

for $(F, \Phi) \in E$, where $\alpha$ and $\beta$ are

$$
\alpha\left(\rho_{0} F, \Phi\right)=\left\{\begin{array}{lc}
0, & \text { if } \rho_{0} F+\frac{1}{2}|\Phi|^{2} \leq 0, \\
+\infty, & \text { otherwise }
\end{array}\right.
$$

and

$$
\beta(F, \Phi)=\left\{\begin{array}{lc}
\langle\bar{c}, F\rangle+\langle\bar{m}, \phi\rangle, & \text { if condition }(P) \text { holds true, } \\
+\infty & \text { otherwise, }
\end{array}\right.
$$

where, for given $F$ and $\Phi$, condition $(P)$ means that there exists $p \in C^{0}(Q)$ and $\phi \in C^{0}\left(Q^{\prime}\right)$ with $\partial_{t} \phi$ and $\nabla_{x} \phi$ in $C^{0}\left(Q^{\prime}\right)$ such that:

$$
F(t, x, a)+\partial_{t} \phi(t, x, a)+p(t, x)=0 \text { and } \Phi(t, x, a)+\nabla_{x} \phi(t, x, a)=0 .
$$

After the adaptation in the definition of $\alpha$ and $\beta$ to the nonhomogeneous case, the rest of the argument follows Brenier's. Indeed, using the Fenchel-Rockafeller Duality Theorem we have

$$
\sup _{(F, \Phi) \in E}\left\{-\alpha_{\rho_{0}}(-F,-\Phi)-\beta_{\rho_{0}}(F, \Phi)\right\}=\inf _{(c, m) \in E^{\prime}}\left\{\alpha_{\rho_{0}}^{*}(c, m)+\beta_{\rho_{0}}^{*}(c, m)\right\},
$$

where $\alpha_{\rho_{0}}^{*}$ and $\beta_{\rho_{0}}^{*}$ denote the Legendre-Fenchel transforms of $\alpha_{\rho_{0}}$ and $\beta_{\rho_{0}}$, respectively.

Computing the convex duals yields:

$$
\alpha_{\rho_{0}}^{*}(c, m)=\frac{1}{2}\left\langle c, \rho_{0}|v|^{2}\right\rangle=\frac{1}{2} \int_{Q^{\prime}}|v|^{2} d c_{\rho_{0}}
$$

and

$$
\beta_{\rho_{0}}^{*}(c, m)=\left\{\begin{array}{lc}
0, & \text { if }\left\langle\bar{c}-c, \partial_{t} \phi+p\right\rangle+\left\langle\bar{m}-m, \nabla_{x} \phi\right\rangle=0, \quad \forall p, \phi, \\
+\infty & \text { otherwise. }
\end{array}\right.
$$

It is easy to check that $\beta_{\rho_{0}}^{*}(c, m)=0$ if and only if the admissible conditions (3.4) and (3.6) are satisfied. Then, the minimum of the action coincides with

$$
\sup _{(F, \Phi) \in E}\left\{-\alpha_{\rho_{0}}(-F,-\Phi)-\beta_{\rho_{0}}(F, \Phi)\right\},
$$

which can be written as

$$
\sup _{p, \phi}\left\{\left\langle c, \rho_{0} \partial_{t} \phi+p\right\rangle+\left\langle m, \rho_{0} \nabla_{x} \phi\right\rangle, \text { such that } \rho_{0} \partial_{t} \phi+\frac{1}{2} \rho_{0}\left|\nabla_{x} \phi\right|^{2}+p \leq 0\right\} \text {. }
$$

Then, we can conclude that for any $\varepsilon>0$, there exist $p_{\varepsilon}(t, x)$ and $\phi_{\varepsilon}(t, x, a)$ satisfying

$$
\rho_{0} \partial_{t} \phi_{\varepsilon}+\frac{1}{2} \rho_{0}\left|\nabla_{x} \phi_{\varepsilon}\right|^{2}+p_{\varepsilon} \leq 0
$$

and

$$
\left.\int_{Q^{\prime}}\left|\rho_{0} \partial_{t} \phi_{\varepsilon}+\frac{1}{2} \rho_{0}\right| \nabla_{x} \phi_{\varepsilon}\right|^{2}+p_{\varepsilon}\left|d c+\int_{Q^{\prime}} \rho_{0}\right| v-\left.\nabla \phi_{\varepsilon}\right|^{2} d c \leq \varepsilon^{2},
$$

where $v$ is the Radon-Nikodým derivative of $m_{\rho_{0}}$ with respect to $c_{\rho_{0}}$, which concludes the proof. 
The proof of (A) and (B) in Theorem 4.1 follows immediately from Lemma 4.2 and Proposition 4.3 above. For the proof of (C), we need a regularity result on $\nabla_{x} \phi_{\varepsilon}$, stated below.

Proposition 4.4. Let $0<\tau<T$ and $Q_{\tau}^{\prime}=[\tau, T-\tau] \times D_{x} \times D_{a}$. If $\omega: D_{x} \rightarrow \mathbb{R}^{2}$ is a smooth divergence-free vector field which is tangent to $\partial D_{x}$, and if $g_{\omega}(\cdot, x)$ : $\mathbb{R} \rightarrow D_{x}$ is the integral curve of $\omega$ beginning on $x$, then, for any optimal solution $\left(c_{\rho_{0}}, m_{\rho_{0}}\right)$ we have:

$$
\int_{Q_{\tau}^{\prime}}\left|\nabla_{x} \phi_{\epsilon}\left(t+\eta, g_{\omega}(\delta, x), a\right)-\nabla_{x} \phi_{\epsilon}(t, x, a)\right|^{2} d c_{\rho_{0}}(t, x, a) \leq\left(\epsilon^{2}+\eta^{2}+\delta^{2}\right) C,
$$

for all $\eta, \delta$ and $\epsilon>0$ sufficiently small, where $C$ depends only on $D, T, \tau$ and $\omega$.

Outline of the proof. Let $\eta>0$ and $\xi=\xi(t) \in C_{0}^{\infty}([0, T])$, with compact support in $(0, T)$ and $\omega: D_{x} \rightarrow D_{x}$ a smooth, divergence-free vector field tangent to $\partial D_{x}$. Denote by $g_{\omega}$ the flow of $\omega$. We introduce $c_{\rho_{0}}^{\eta}=c_{\rho_{0}}^{\eta}(t, x, a)=c_{\rho_{0}}(t+\eta \xi(t), x, a)$, $v_{\rho_{0}}^{\eta}=v(t+\eta \xi(t), x, a)\left(1+\eta \xi^{\prime}(t)\right)$ and we introduce the measures $c_{\rho_{0}}^{\eta, \delta}$ and $m_{\rho_{0}}^{\eta, \delta}$ by the identities:

$$
\begin{gathered}
\int_{Q^{\prime}} f(t, x, a) d c_{\rho_{0}}^{\eta, \delta}(t, x, a)=\int_{Q^{\prime}} f\left(t, g_{\omega}(\delta \xi(t), x), a\right) d c_{\rho_{0}}^{\eta, \delta}(t, x, a), \\
\int_{Q^{\prime}} f(t, x, a) d m_{\rho_{0}}^{\eta, \delta}(t, x, a) \\
=\int_{Q^{\prime}} f\left(t, g_{\omega}(\delta \xi(t), x), a\right)\left(\partial_{t}+v^{\eta}(t, x, a) \cdot \nabla_{x}\right)\left(g_{\omega}(\delta \xi(t), x)\right) d c_{\rho_{0}}^{\eta}(t, x, a),
\end{gathered}
$$

valid for all $f \in C\left(Q^{\prime}\right)$.

Using (4.4) and (4.5) and implementing the identities above one can derive the inequality

$$
\begin{gathered}
\int_{Q^{\prime}} p_{\epsilon}(t, x) d c^{\eta, \delta}(t, x, a)-\int_{Q^{\prime}} p_{\epsilon}(t, x) d c(t, x, a) \\
+\frac{1}{2} \int_{Q^{\prime}}\left|\left(\partial_{t}+v^{\eta}(t, x, a) \cdot \nabla_{x}\right)\left(g_{\omega}(\delta \xi(t), x)\right)-\nabla_{x} \phi_{\epsilon}\left(t, g_{\omega}(\delta \xi(t), x), a\right)\right|^{2} d c_{\rho_{0}}^{\eta}(t, x, a) \\
\leq \epsilon^{2}+\frac{1}{2} \int_{Q^{\prime}}\left|\left(\partial_{t}+v^{\eta}(t, x, a) \cdot \nabla_{x}\right)\left(g_{\omega}(\delta \xi(t), x)\right)\right|^{2} d c_{\rho_{0}}^{\eta}(t, x, a) \\
-\frac{1}{2} \int_{Q^{\prime}}|v(t, x, a)|^{2} d c_{\rho_{0}}(t, x, a) .
\end{gathered}
$$


Using the inequality above we obtain (4.7), (4.8) and (4.9). To obtain (4.6), we first set $\delta=0$ in (4.10), and then we use the fact that

$$
\int_{Q^{\prime}} p_{\varepsilon}(t, x) d c^{\eta}(t, x, a)-\int_{Q^{\prime}} p_{\varepsilon}(t, x) d c(t, x, a)=0,
$$

and we take $\varepsilon \rightarrow 0$ to obtain:

$$
0 \leq \int_{Q^{\prime}}\left|v^{\eta}\right|^{2} d c_{\rho_{0}}^{\eta}(t, x, a)-\int_{Q^{\prime}}|v|^{2} d c_{\rho_{0}}(t, x, a) .
$$

Set

$$
\mathbb{K}(t) \equiv \frac{1}{2} \int_{D_{x} \times D_{a}}|v(t, x, a)|^{2} c_{\rho_{0}}(t, x, a) d x d a,
$$

so that (4.11) becomes

$$
\begin{aligned}
\int_{0}^{T} \mathbb{K}(t) d t \leq \int_{0}^{T} & \left(\mathbb{K}(t+\eta \xi(t))\left(1+\eta \xi^{\prime}(t)\right)+\mathbb{K}(t+\eta \xi(t)) \eta \xi^{\prime}(t)\right. \\
& \left.+\mathbb{K}(t+\eta \xi(t)) \eta^{2}\left(\xi^{\prime}(t)\right)^{2}\right) d t
\end{aligned}
$$

The first term on the right-hand side of (4.12) is none other than $\int_{0}^{T} \mathbb{K}(t) d t$. Hence, dividing (4.12) by $\eta$ we find

$$
\int_{0}^{T} \mathbb{K}(t+\eta \xi(t)) \xi^{\prime}(t)+\mathbb{K}(t+\eta \xi(t)) \eta\left(\xi^{\prime}(t)\right)^{2} d t \geq 0 .
$$

Taking the limit as $\eta \rightarrow 0$ we obtain $\int_{0}^{T} \mathbb{K}(t) \xi^{\prime}(t) d t \geq 0$. Using $-\xi$ in place of $\xi$ we find the opposite inequality and hence $\int_{0}^{T} \mathbb{K}(t) \xi^{\prime}(t) d t=0$, which yields (4.6). This concludes the proof.

Item (C) of Theorem 4.1 follows immediately from Proposition 4.4, To conclude our discussion of the proof of Theorem 4.1, we are left with the construction of $\nabla_{x} p$ and its uniqueness. This is the most delicate part of Brenier's argument. Its proof can be naturally divided into five steps as follows:

(1) the family $p_{\varepsilon}$ is bounded in the sense of distributions in the interior of $Q$;

(2) the derivation of a discretized Euler-Lagrange equation for the relaxed problem;

(3) convergence in distributions of the family $\left\{\nabla p_{\varepsilon}\right\}$ to a unique limit $\nabla p$;

(4) proof that the distribution $\nabla p$ is actually a locally bounded measure in $Q$;

(5) derivation of (4.2).

The proof of (1) follows from inequality (4.10) with $\eta=0$, using a suitable change of variables to transform integrals with respect to $d c$ to integrals with respect to the Lebesgue measure.

For the proofs of (2) and (3), we use the estimates obtained in Proposition 4.4 to derive an approximate version of the Euler-Lagrange equation of the relaxed problem of the form:

$$
\mid \int_{Q^{\prime}} \frac{1}{2 \delta}\left[p_{\epsilon}\left(t, g_{\omega}(\delta, x)\right)-p_{\epsilon}\left(t, g_{\omega}(-\delta, x)\right)\right] f(t, x, a) d c(t, x, a)
$$

$$
-\int_{Q^{\prime}} v(t, x, a)\left(\partial_{t}+v(t, x, a) \cdot \nabla_{x}\right)(\omega(x) f(t, x, a)) d c_{\rho_{0}}(t, x, a) \mid \leq C_{f} \frac{\left(\delta^{2}+\epsilon^{2}\right)}{\delta},
$$


where $f \in C_{0}^{\infty}\left(Q^{\prime}\right)$ and $\omega: D_{x} \rightarrow D_{x}$ is a smooth, divergence-free vector field tangent to $\partial D$. We choose $f \in C^{1}\left(Q^{\prime}\right)$ vanishing in a neighborhood of 0 and $T$, which is independent of $a$, i.e., $f(t, x, a) \equiv f(t, x)$. The inequality (4.13) becomes:

$$
\begin{gathered}
\mid \int_{0}^{T} \int_{D_{y}} \frac{1}{2 \delta}\left[f\left(t, g_{\omega}(-\delta, y)\right)-f\left(t, g_{\omega}(\delta, y)\right)\right] p_{\epsilon}(t, y) d y d t \\
-\int_{Q^{\prime}} v(t, x, a)\left(\partial_{t}+v(t, x, a) \cdot \nabla_{x}\right)(\omega(x) f(t, x)) d c_{\rho_{0}}(t, x, a) \mid \leq C_{f} \frac{\left(\delta^{2}+\epsilon^{2}\right)}{\delta} .
\end{gathered}
$$

Since $f$ does not depend on $a, C_{f}$ only depends on $f$, through its support in $t$, and on its norm

$$
|||f| \|=\sup _{Q}\left(|f|+\left|\nabla_{x} f\right|\right)+\left(\int_{Q}\left|\partial_{t} f\right|^{2} d t d x\right)^{\frac{1}{2}} .
$$

As $\left(p_{\varepsilon}\right)$ is bounded in distributions, we can find a converging subsequence in $\mathcal{D}^{\prime}$. Passing to the limit along this subsequence first, and then taking $\delta \rightarrow 0$ in (4.14), we can derive:

$$
-\nabla_{x} p(t, x)=\partial_{t} \int_{D_{a}} v(t, x, a) c_{\rho_{0}}(t, x, d a)+\nabla_{x} \cdot \int_{D_{a}}(v \otimes v)(t, x, a) c_{\rho_{0}}(t, x, d a) .
$$

Uniqueness follows from the fact that the pair $\left(c_{\rho_{0}}, m_{\rho_{0}}\right)$ is an arbitrary optimal solution.

Next we examine the proof of (4). Let $\xi:[0, T] \longrightarrow[0,1]$ be a cutoff such that $\xi(t)=0$ near $t=0, T$ and $\xi(t)=1$ outside a neighborhood of $t=0, T$. Consider also $\eta>0$ and $\delta>0$ small enough, so that $\xi(t-\theta \eta)$ vanishes near $t=0, T$, for all $\theta \in[-1,1]$. Using the estimates in Proposition 4.4, we conclude that there exists $\tau>0$, sufficiently small and $C>0$, depending on $D_{x}, T, \tau$ and $\omega$ such that

$$
\int_{Q_{\tau}}\left|\int_{0}^{1} p_{\varepsilon}\left(t+\eta \theta, g_{\omega}(\delta, x)\right)-p_{\varepsilon}(t+\eta \theta, x) d \theta\right| d x d t \leq\left(1+\frac{\delta}{\eta}\right)(\eta+\delta+\varepsilon) C,
$$

where $0<\tau<T$ and $Q_{\tau}=[\tau, T-\tau] \times D_{x}$. Taking $\varepsilon \rightarrow 0$ and setting $\eta=\delta$ above we conclude that $p$ satisfies

$$
\frac{1}{\delta} \int_{Q_{\tau+\delta \theta}} p(s, x)\left(f\left(t-\delta \theta, g_{\omega}(-\delta, x)\right)-f(t-\delta \theta, x)\right) d x d t d \theta \leq C \sup |f|,
$$

for any $f(t, x) \geq 0$ with compact support in $0<t<T$.

Taking first $\delta \rightarrow 0$ and then $\theta \rightarrow 0$ in this inequality yields

$$
\langle\nabla p(t, x) \omega(x), f(t, x)\rangle \leq C \sup |f|,
$$

where $C$ depends on $D_{x}, T, \omega$ and in the support of $f$, which proves that $\nabla p$ is a locally bounded measure.

Finally, we examine (5). The point of departure is (4.13), passing to the limit $\varepsilon \leq \delta \rightarrow 0$. This is complicated, since we only know that

$$
\int_{Q} \frac{1}{2 \delta}\left[p_{\varepsilon}\left(t, g_{\omega}(\delta, x)\right)-p_{\varepsilon}\left(t, g_{\omega}(-\delta, x)\right)\right] f(t, x) d t d x \rightarrow \int_{Q} \nabla_{x} p(t, x) f(t, x) d t d x,
$$

when $f \in C_{0}^{\infty}(Q)$, and the first integral in (4.13) involves the measure $c$.

To deal with this difficulty, we begin with the fact that

$$
p_{\varepsilon}\left(t, g_{\omega}(\delta, x)\right)-p_{\varepsilon}\left(t, g_{\omega}(-\delta, x)\right) \rightarrow p\left(t, g_{\omega}(\delta, x)\right)-p\left(t, g_{\omega}(-\delta, x)\right),
$$


$\varepsilon \rightarrow 0$, weakly in $X^{\prime}$, where $X$ is the completion of $C_{0}^{\infty}(Q)$ with respect to the norm

$$
\|f\|=\sup _{Q}\left(|f|+\left|\nabla_{x} f\right|\right)+\left(\int_{Q}\left|\partial_{t} f\right|^{2} d t d x\right)^{\frac{1}{2}} .
$$

Fix $e$ a vector in the unit ball in $\mathbb{R}^{n}$. We fix a function $f \in C_{0}^{\infty}\left(Q^{\prime}\right)$ and we choose a vector field $\omega$, divergence-free and tangent to $\partial D_{x}$ such that, for those $(t, x, a)$ where $f(t, x, a) \neq 0$, we have $\omega(t, x, a)=e$. Fix $t$ and $a$, and let $x \in D_{x}$ such that $f(t, x, a) \neq 0$. Then, for $\delta$ sufficiently small, $g_{\omega}(\delta, x)=x \pm \delta$. Hence, using $\omega$ in (4.13) we get

$$
\begin{gathered}
\mid \int_{Q^{\prime}} \frac{1}{2 \delta}\left(p_{\epsilon}(t, x+\delta e)-p_{\epsilon}(t, x-\delta e)\right) f(t, x, a) d c(t, x, a) \\
-\int_{Q^{\prime}} v(t, x, a)\left(\partial_{t}+v(t, x, a) \cdot \nabla_{x}\right)(f(t, x, a) e) d c_{\rho_{0}}(t, x, a) \mid \leq \frac{1}{\delta}\left(\delta^{2}+\epsilon^{2}\right) C_{f}
\end{gathered}
$$

where $C_{f}$ depends on $T, D_{x}$, in $\omega$, and it depends on $f$ through $\||f|\|$ and the size of its temporal support only.

Let $\gamma$ be a Friedrichs mollifier in $\mathbb{R}^{n}$, centered at the origin, with support in the ball of radius $\frac{1}{2}$. Fix a vector $\bar{e} \in B_{\frac{1}{2}}(0)$.

For each $y \in B_{\frac{1}{2}}(0)$, we rewrite (4.16) in the form

$$
\begin{gathered}
\mid \int_{Q^{\prime}} \frac{1}{2 \delta}\left(p_{\epsilon}(t, x+\delta(\bar{e}+y))-p_{\epsilon}(t, x-\delta(\bar{e}+y))\right) f(t, x, a) d c(t, x, a) \\
-\int_{Q^{\prime}} v(t, x, a)\left(\partial_{t}+v(t, x, a) \cdot \nabla_{x}\right)(f(t, x, a)(\bar{e}+y)) d c_{\rho_{0}}(t, x, a) \mid \leq \frac{1}{\delta}\left(\delta^{2}+\epsilon^{2}\right) C_{f} .
\end{gathered}
$$

A simple calculation, using the circular symmetry of $\gamma$, yields

$$
\begin{gathered}
\mid \int_{Q} \frac{1}{2 \delta}\left(p_{\epsilon}(t, x+\delta \bar{e})-p_{\epsilon}(t, x-\delta \bar{e})\right) f_{c, \delta, \gamma}(t, x) d t d x \\
-\int_{Q^{\prime}} v(t, x, a) \bar{e}\left(\partial_{t}+v(t, x, a) \cdot \nabla_{x}\right) f(t, x, a) d c_{\rho_{0}}(t, x, a) d y \mid \leq \frac{1}{\delta}\left(\delta^{2}+\epsilon^{2}\right) C_{f}
\end{gathered}
$$

where

$$
f_{c, \delta, \gamma}(t, x)=\int_{B_{\frac{1}{2}}(0)} \int_{D_{a}} f(t, x-\delta y, a) \gamma(y) c(t, x-\delta y, d a) d y \in X
$$

Hence, for each fixed $\delta$, we can pass to the limit $\epsilon \rightarrow 0$ in (4.17), using the weak-* convergence $p_{\epsilon}\left(t, g_{\omega}(\delta, x)\right)-p_{\epsilon}\left(t, g_{\omega}(-\delta, x)\right) \longrightarrow p\left(t, g_{\omega}(\delta, x)\right)-p\left(t, g_{\omega}(-\delta, x)\right)$ weak* to obtain

$$
\begin{gathered}
\mid \int_{Q} \frac{1}{2 \delta}(p(t, x+\delta \bar{e})-p(t, x-\delta \bar{e})) f_{c, \delta, \gamma}(t, x) d t d x \\
-\int_{Q^{\prime}} v(t, x, a) \bar{e}\left(\partial_{t} f(t, x, a)+v(t, x, a) \nabla_{x} f(t, x, a)\right) d c_{\rho_{0}}(t, x, a) \mid \leq \delta C_{f} .
\end{gathered}
$$


Next we perform the change of variables $z=x+\delta \bar{e}$ and $z=x-\delta \bar{e}$ to get

$$
\begin{gathered}
\mid \int_{Q} p(t, x) \bar{e}\left(-\nabla_{x} \int_{-\frac{1}{2}}^{\frac{1}{2}} f_{c, \delta, \omega}(t, x-2 \theta \delta \bar{e}) d \theta\right) d t d x \\
-\int_{Q^{\prime}} v(t, x, a) \bar{e}\left(\partial_{t} f(t, x, a)+v(t, x, a) \nabla_{x} f(t, x, a)\right) d c_{\rho_{0}}(t, x, a) \mid \leq \delta C_{f} .
\end{gathered}
$$

As

$$
\begin{aligned}
& \int_{Q} p(t, x) \bar{e}(-\left.\nabla_{x} \int_{-\frac{1}{2}}^{\frac{1}{2}} f_{c, \delta, \omega}(t, x-2 \theta \delta \bar{e}) d \theta\right) d t d x \\
&=\left\langle\nabla_{x} p(t, x) \bar{e}, \int_{-\frac{1}{2}}^{\frac{1}{2}} f_{c, \delta, \omega}(t, x-2 \theta \delta \bar{e}) d \theta\right\rangle
\end{aligned}
$$

we conclude that

$$
\begin{gathered}
\mid\left\langle\nabla_{x} p(t, x) \bar{e}, \int_{-\frac{1}{2}}^{\frac{1}{2}} f_{c, \delta, \omega}(t, x-2 \theta \delta \bar{e}) d \theta\right\rangle \\
-\int_{Q^{\prime}} v(t, x, a) \bar{e}\left(\partial_{t} f(t, x, a)+v(t, x, a) \nabla_{x} f(t, x, a)\right) d c_{\rho_{0}}(t, x, a) \mid \leq \delta C_{f} .
\end{gathered}
$$

Next we introduce

$$
c_{\delta, e, \gamma}(t, x, a)=\int_{-\frac{1}{2}}^{\frac{1}{2}} \int_{B_{\frac{1}{2}}(0)} c(t, x-2 \theta \delta \bar{e}-\delta y, a) \gamma(y) d y d \theta,
$$

which is a mollification of $c$, continuous in $t$ and smooth in $x$, taking values on the space of Borel probabilities in $a$.

Using the definition of $f_{c, \delta, \gamma}$ and of $c_{\delta, e, \gamma}$, we conclude that

$$
\left|\int_{-\frac{1}{2}}^{\frac{1}{2}} f_{c, \delta, \gamma}(t, x-2 \theta \delta \bar{e}) d \theta-\int_{D_{a}} f(t, x, a) c_{\delta, \bar{e}, \gamma}(t, x, d a)\right| \leq \delta C_{f}
$$

and hence, from (4.18) we conclude that

$$
\begin{gathered}
\mid\left\langle\nabla_{x} p(t, x) \bar{e}, \int_{D_{a}} f(t, x, a) c_{\delta, \bar{e}, \gamma}(t, x, d a)\right\rangle \\
-\int_{Q^{\prime}} v(t, x, a) \bar{e}\left(\partial_{t} f(t, x, a)+v(t, x, a) \nabla_{x} f(t, x, a)\right) d c_{\rho_{0}}(t, x, a) \mid \leq \delta C_{f} .
\end{gathered}
$$

Since $\nabla p$ is a locally bounded measure in $Q$ and $c_{\delta, e, \gamma}(t, x, a)$ is a probability measure in $a$, we have

$$
\left|\left\langle\nabla_{x} p(t, x) \bar{e}, \int_{D_{a}} f(t, x, a) c_{\delta, e, \gamma}(t, x, d a)\right\rangle\right| \leq\left\langle\left|\nabla_{x} p(t, x) \bar{e}\right| \sup _{a \in D_{a}}|f(t, x, a)|\right\rangle .
$$

Therefore, $c_{\delta, \bar{e}, \gamma}$ may be understood as a bounded subset of $L^{\infty}\left(|\nabla p|, C\left(D_{a}\right)\right)$, which is the dual of $L^{1}\left(|\nabla p|, C\left(D_{a}\right)\right)$. 
Hence, since

$$
\begin{gathered}
\mid\left\langle\nabla p(t, x) \bar{e}, \int_{D_{a}} f(t, x, a) c_{\delta, \bar{e}, \gamma}(t, x, d a)\right\rangle \\
-\int_{Q^{\prime}} v(t, x, a) \bar{e}\left(\partial_{t} f(t, x, a)+v(t, x, a) \nabla_{x} f(t, x, a)\right) d c_{\rho_{0}}(t, x, a) \mid \leq \delta C_{f},
\end{gathered}
$$

for each fixed $\bar{e}$ and $\gamma$, there exists a unique limit when $\delta \rightarrow 0^{+}$, which we denote by $c_{\bar{e}, \gamma}$, satisfying

$$
\begin{gathered}
\left\langle\nabla p(t, x) \bar{e}, \int_{D_{a}} f(t, x, a) c_{\delta, e, \gamma}(t, x, d a)\right\rangle \\
=\int_{Q^{\prime}} v(t, x, a) \bar{e}\left(\partial_{t} f(t, x, a)+v(t, x, a) \nabla_{x} f(t, x, a)\right) d c_{\rho_{0}}(t, x, a) .
\end{gathered}
$$

Since the right-hand side does not depend on $\gamma$ and it depends linearly on $\bar{e}$, the limit $c_{\bar{e}, \gamma}$ cannot depend on $\gamma$ and $\bar{e}$. Therefore, the limit in $\delta$ may be denoted by $\underline{c}$ and it satisfies

$$
\begin{gathered}
\left\langle\nabla p(t, x) \bar{e}, \int_{D_{a}} f(t, x, a) \underline{c}(t, x, d a)\right\rangle \\
=\int_{Q^{\prime}} v(t, x, a) \bar{e}\left(\partial_{t} f(t, x, a)+v(t, x, a) \nabla_{x} f(t, x, a)\right) d c_{\rho_{0}}(t, x, a),
\end{gathered}
$$

where

$$
\underline{c}=\lim _{\delta \longrightarrow 0} \int_{-\frac{1}{2}}^{\frac{1}{2}} \int_{B_{\frac{1}{2}}(0)} c(t, x-2 \theta \delta \bar{e}-\delta y, a) \gamma(y) d y d \theta .
$$

Therefore,

$$
\partial_{t}\left(c_{\rho_{0}} v\right)+\nabla_{x} \cdot\left(c_{\rho_{0}} v \otimes v\right)+\underline{c} \nabla p=0
$$

is the Euler-Lagrange equation associated with the relaxed problem. This concludes our discussion of Brenier's proof and our adaptation to nonhomogeneous flows. For details, see [12].

\section{Consistency of the Relaxed solutions with the solutions OF THE EULER EQUATIONS}

We would like to regard the relaxed Euler equations introduced in the previous section as a generalized formulation of the incompressible Euler equations. In order to justify this point of view it is necessary to establish consistency; that is the main purpose of this section.

We will prove two results. First, we will show that, if the solution of the relaxed system has a particular structure, then this solution gives rise to a (classical) weak solution of the Euler equations. We observe that this result is new even for the homogeneous case. Next, we show that smooth solutions of the Euler equations give rise to solutions of the relaxed Euler equations. This second result is an extension of the analogous statement in the homogeneous case, proved by Brenier; see [Theorem 2.4, 7]]. As was done for the homogeneous case in [Theorem 2.4 and Proposition 3.2, 7] we also show that, for small enough $T$, there is a unique minimum of the action (2.3); the size of $T$ is controlled by the maximum eigenvalue of the Hessian matrix of the pressure. 
Fix $h \in G(D)$ and let $g \in C^{2}(Q)$ be a path such that, for each $t, g(t, \cdot) \in G(D)$, $g(0, x)=x$ and $g(T, x)=h(x)$. Note that, for dimension $N \geq 3$, any orientationpreserving diffeomorphism has a smooth path connecting it to the identity, whereas, for $N=2$, the existence of such a path is an additional hypothesis on $h$; see [14, 15].

We begin with a technical lemma.

Lemma 5.1. Let $f \in\left(C_{0}^{1}(Q)\right)^{N}$. Then there exists $\varphi \in C_{0}^{1}\left(Q^{\prime}\right)$ such that, for all $t \in[0, T]$ and $a \in[0,1]^{N}$, we have

$$
\nabla_{x} \varphi(t, g(t, a), a)=f(t, a) .
$$

Proof. Fix $f \in\left(C_{0}^{1}(Q)\right)^{2}$. The proof involves an explicit construction.

Set

$$
\Sigma=\left\{(t, x, a) \in Q^{\prime} \mid x=g(t, a)\right\}
$$

and let $\mu:[0, T] \times[0,1]^{N} \rightarrow \Sigma$ be given by $\mu(t, a)=(t, g(t, a), a)$. Clearly, $\mu$ is a parameterization of the surface $\Sigma$.

Consider a tubular neighborhood $\Omega \subset \mathbb{R}^{2 N+1}$ of $\Sigma$ and a set of normal vectors to $\Sigma, \widehat{n}_{i}, i=1, \ldots, N$. We choose the $\widehat{n}_{i}$ explicitly by

$$
\widehat{n}_{i}(t, a)=\left(-\frac{\partial g_{i}}{\partial t}(t, a), e_{i},-\frac{\partial g_{i}}{\partial a_{1}}(t, a), \ldots,-\frac{\partial g_{i}}{\partial a_{N}}(t, a)\right),
$$

where $e_{i}$ is the vector with $j$-th component $\delta_{i j}$.

For sufficiently small $\varepsilon>0$, we define $Z:[0, T] \times[-\varepsilon, \varepsilon]^{N} \times[0,1]^{N} \rightarrow \Omega$ to be the surjective map given by

$$
Z\left(t, r_{1}, \ldots, r_{N}, a\right)=\mu(t, a)+\sum_{i} r_{i} \widehat{n}_{i}(t, a) .
$$

Denote by $\omega:[0, T] \times[0,1]^{N} \rightarrow \mathbb{R}^{2 N+1}$ a given vector field, to be chosen later.

Let $\psi:[0, T] \times[-\varepsilon, \varepsilon]^{N} \times[0,1]^{N} \rightarrow \mathbb{R}$ denote the function

$$
\psi\left(t, r_{1}, \ldots, r_{N}, a\right)=\sum_{i} r_{i} \widehat{n}_{i}(t, a) \cdot \omega(t, a) .
$$

Set $\varphi: \Omega \rightarrow \mathbb{R}$ so that

$$
\varphi(t, x, a)=\left(\psi \circ Z^{-1}\right)(t, x, a) .
$$

Hence, $\nabla \varphi=\left(\nabla \psi \circ Z^{-1}\right)^{T} D Z^{-1}$.

Note that

$$
\left(\nabla \psi \circ Z^{-1}\right)_{\left.\right|_{\Sigma}}^{T}=\left(0, \widehat{n}_{1} \cdot \omega, \ldots, \widehat{n}_{N} \cdot \omega, 0\right)
$$

and that, if we write $\partial_{t} g$ as a row vector, and we denote by $D_{a} g$ the Jacobian matrix of $g$ with respect to the $a$ variables while $\mathbb{I}_{N}$ is the $N \times N$ identity, we can write

$$
D Z(t, 0, a)=\left(\begin{array}{ccc}
1 & -\partial_{t} g & 0 \\
\left(\partial_{t} g\right)^{t} & \mathbb{I}_{N} & D_{a} g \\
0 & -\left(D_{a} g\right)^{t} & \mathbb{I}_{N}
\end{array}\right) .
$$

First we observe that $M \equiv D Z(t, 0, a)$ is invertible. This follows from the fact that $M$ is of the form $\mathbb{I}+A$ with $A$ antisymmetric.

Next, let $P=M^{-1}=\left(p_{i j}\right)$, with $i, j=1, \ldots, 2 N+1$. We wish to prove that the $N \times N$ minor $\left(p_{i j}\right), 2 \leq i, j \leq N+1$ is invertible. We denote this $N \times N$ minor by $P_{0}$. 
First, consider the orthogonal $2 N+1 \times 2 N+1$ matrix $O$, whose first row is the vector $e_{2 N+1}$, followed by $e_{1}, \ldots, e_{2 N}$. Conjugation with $O$ places the first row and the first column as the last row and column. More precisely,

$$
\widetilde{M} \equiv O^{-1} M O=\left(\begin{array}{ccc}
\mathbb{I}_{N} & D_{a} g & \left(\partial_{t} g\right)^{t} \\
\left(-D_{a} g\right)^{t} & \mathbb{I}_{N} & 0 \\
-\partial_{t} g & 0 & 1,
\end{array}\right) \equiv\left(\begin{array}{cc}
\mathbb{I}_{N} & D \\
-D^{t} & \mathbb{I}_{N+1}
\end{array}\right),
$$

with $D$ the $N \times(N+1)$ matrix $\left[D_{a} g\left(\partial_{t} g\right)^{t}\right]$. Next, note that

$$
\widetilde{M}^{-1}=\left(O^{-1} M O\right)^{-1}=O^{-1} M^{-1} O \equiv \widetilde{P},
$$

so that $P_{0}$ is now the top-left $N \times N$ minor of $\widetilde{P}$.

We write

$$
\widetilde{P}=\left(\begin{array}{cc}
P_{0} & X \\
Y & Z
\end{array}\right)
$$

where $X$ is an $N \times(N+1)$ matrix. From the fact that $\widetilde{P} \widetilde{M}=\mathbb{I}_{2 N+1}$, we deduce that

$$
P_{0}-X D^{t}=\mathbb{I}_{N}
$$

and

$$
P_{0} D+X=0_{N \times(N+1)} .
$$

Hence $P_{0}\left(\mathbb{I}_{N}+D D^{t}\right)=\mathbb{I}_{N}$, which implies $P_{0}$ is invertible, as desired.

We now choose $\omega$ conveniently; since $P_{0}$ is invertible and the vectors $\widehat{n}_{i}$ are linearly independent, it is possible to find a vector $w$ such that:

$$
P_{0}\left(\begin{array}{c}
\widehat{n}_{1} \cdot w \\
\cdots \\
\widehat{n}_{N} \cdot w
\end{array}\right)=\left(\begin{array}{c}
f_{1} \\
\cdots \\
f_{N}
\end{array}\right) .
$$

Therefore, in view of (5.5), given $f \in\left(C_{0}^{1}\left([0,1] \times[0,1]^{2}\right)\right)^{N}$, where $f(t, a)=$ $\left(f_{1}(t, a), \ldots, f_{N}(t, a)\right)$, we have exhibited $\varphi \in C_{0}^{1}\left(Q^{\prime}\right)$ such that $\nabla_{x} \varphi(t, g(t, a), a)=$ $\left(f_{1}(t, a), \ldots, f_{N}(t, a)\right)$.

Let $h \in G(D) \cap C^{\infty}(D)$ and consider a path $g \in C^{\infty}(Q)$ such that $g(t, \cdot) \in G(D)$, $g(0, x)=x$ and $g(T, x)=h(x)$. For each such $g$ we introduce

$$
Y_{t}=\left\{(x, a) \in D_{x} \times D_{a} \mid x=g(t, a)\right\} .
$$

Consider the measure $c$ given by

$$
c(t, x, a)=\delta_{(t, g(t, a), a)} .
$$

Let $\nabla_{x} p \in \mathcal{D}^{\prime}(Q)$. We wish to consider the restriction of $\nabla_{x} p$ to $Y_{t}$. According to an elementary result in microlocal analysis, see [10], such a restriction exists if the conormal bundle of $Y_{t}$ does not intercept the wave-front set of $\nabla_{x} p$. It is straightforward to verify this condition, using that $p$ is independent of $a$ and $g$ is a smooth diffeomorphism. We will write this restriction $\left.\nabla_{x} p\right|_{Y_{t}}$ as $\nabla_{x} p(t, g(t, a))$. We define the product $c \nabla_{x} p$ as a distribution in $\mathcal{D}^{\prime}\left(Q^{\prime}\right)$ through the identity

$$
\begin{aligned}
\int_{Q^{\prime}} c(t, x, a) & \nabla_{x} p(t, x) \phi(t, x, a) d t d x d a \\
= & \int_{0}^{T} \int_{D_{a}} \nabla_{x} p(t, g(t, a)) \phi(t, g(t, a), a) d a d t, \quad \forall \phi \in C_{0}^{\infty}\left(Q^{\prime}\right) .
\end{aligned}
$$


We are now ready to state and prove our first consistency result.

Theorem 5.2. Let $h \in G(D) \cap C^{\infty}(D)$ and $g \in C^{\infty}(Q)$ be such that $g(t, \cdot) \in$ $G(D), g(0, x)=x$ and $g(T, x)=h(x)$. Consider an optimal solution $\left(c_{\rho_{0}}, m_{\rho_{0}}\right)$ as obtained in Theorem 4.1. Assume that $m_{\rho_{0}}=c_{\rho_{0}} v$ for some $v \in C^{1}\left(Q^{\prime}\right)$. Suppose additionally that

$$
c_{\rho_{0}}(t, x, a)=\rho_{0}(a) \delta_{(t, g(t, a), a)} .
$$

Define

$$
u=u(t, x) \equiv v\left(t, x, g^{-1}(t, x)\right)
$$

Then, for every $t, x$,

$$
\partial_{t} g(t, x)=u(t, g(t, x))
$$

and $u=u(t, x)$ is a weak solution of the nonhomogeneous Euler equations (2.1) with density $\rho=\rho(t, g(t, a))=\rho_{0}(a)$.

Proof. We begin by proving (5.13). Let $\varphi \in C_{0}^{1}\left(Q^{\prime}\right)$. From (3.5) and (5.11) it follows that

$$
\begin{aligned}
0 & =\left\langle\partial_{t} c_{\rho_{0}}+\nabla_{x} \cdot m_{\rho_{0}}, \varphi\right\rangle \\
& =-\int_{[0, T] \times D} \rho_{0}(a)\left[\partial_{t} \varphi(t, g(t, a), a)+v(t, g(t, a), a) \cdot \nabla_{x} \varphi(t, g(t, a), a)\right] d t d a \\
& =\int_{[0, T] \times D} \rho_{0}(a)\left[\partial_{t} g(t, a)-v(t, g(t, a), a)\right] \cdot \nabla_{x} \varphi(t, g(t, a), a) d t d a,
\end{aligned}
$$

since $\partial_{t} \varphi(t, g(t, a), a)=\partial_{t}(\varphi(t, g(t, a), a))-\partial_{t} g(t, a) \nabla_{x} \varphi(t, g(t, a), a)$.

In view of Lemma [5.1] any test function $f \in\left(C_{0}^{1}\left([0, T] \times[0,1]^{N}\right)\right)^{2}$ can be written as $f=f(t, a)=\nabla_{x} \varphi(t, g(t, a), a)$ for some $\varphi \in C_{0}^{1}\left(Q^{\prime}\right)$. Hence

$$
\partial_{t} g(t, a)=v(t, g(t, a), a)
$$

(5.13) now follows from the definition of $u$ in (5.12).

That $u$ is divergence-free follows immediately from the transport theorem together with the fact that

$$
\left\{\begin{array}{l}
u(t, g(t, a))=\partial_{t} g(t, a) \\
g(0, a)=a \\
g \in G(D)
\end{array}\right.
$$

Our next step is to verify that $u$ satisfies the momentum equation in the incompressible Euler system. Our point of departure is (4.2); we begin by examining the first two terms in equation (4.2).

Let $\phi \in\left(C_{0}^{\infty}\left(Q^{\prime}\right)\right)^{2}$. We have

$$
\begin{gathered}
\left\langle\partial_{t} m_{\rho_{0}}, \phi\right\rangle=-\int_{[0, T] \times D} \rho_{0}(a) v(t, g(t, a), a) \partial_{t} \phi(t, g(t, a), a) d t d a \\
=\int_{[0, T] \times D} \rho_{0}(a)\left[\partial_{t}[v(t, g(t, a), a)] \phi(t, g(t, a), a)+\left(v D_{x} \phi v\right)(t, g(t, a), a)\right] d t d a
\end{gathered}
$$

where we have integrated by parts in time after using that $\partial_{t} \phi(t, g(t, a), a)=$ $\partial_{t}[\phi(t, g(t, a), a)]-D_{x} \phi(t, g(t, a), a) \partial_{t} g(t, a)$ and that $\partial_{t} g(t, a)=v(t, g(t, a), a)$. 
Hence we find

$$
\left.\left\langle\partial_{t} m_{\rho_{0}}, \phi\right\rangle=\int_{[0, T] \times D} \rho_{0}(a)\left[\phi \cdot \partial_{t} v+\phi D_{x} v v+v D_{x} \phi v\right)\right]_{\left.\right|_{(t, g(t, a), a)}} d t d a .
$$

Clearly we also have

$$
\left\langle\operatorname{div}_{x}\left(v \otimes m_{\rho_{0}}\right), \phi\right\rangle=-\int_{[0, T] \times D} \rho_{0}(a)\left(v D_{x} \phi v\right)_{\left.\right|_{(t, g(t, a), a)}} d t d a .
$$

Adding (5.14) and (5.15) and effecting the change of variables $(t, g(t, a), a) \mapsto$ $\left(t, x, g^{-1}(t, x)\right)$ we find

$$
\left\langle\partial_{t}\left(m_{\rho_{0}}\right)+\operatorname{div}_{x}\left(v \otimes m_{\rho_{0}}\right), \phi\right\rangle=\int_{[0, T] \times D} \rho(t, x)\left[\phi \cdot \partial_{t} v+\phi D_{x} v v\right]_{\left.\right|_{\left(t, x, g^{-1}(t, x)\right)}} d t d x .
$$

Now, let us observe that

$$
\left(\phi D_{x} v v\right)_{\left.\right|_{\left(t, x, g^{-1}(t, x)\right)}}=\phi D_{x}\left[v\left(t, x, g^{-1}(t, x)\right)\right] v-\phi\left[\left(D_{a} v\right)_{\left.\right|_{\left(t, x, g^{-1}(t, x)\right)}} D_{x} g^{-1}(t, x)\right] v
$$

and

(5.18)

$\phi D_{x}\left[v\left(t, x, g^{-1}(t, x)\right)\right] v=\phi \cdot \operatorname{div}_{x}\left[(v \otimes v)\left(t, x, g^{-1}(t, x)\right)\right]-\phi \operatorname{div}_{x}\left[v\left(t, x, g^{-1}(t, x)\right)\right] \cdot v$.

(We have indicated evaluations only to distinguish between differentiating the composite function and evaluating the derivative.)

We also note that

$$
\left(\phi \cdot \partial_{t} v\right)_{\left.\right|_{\left(t, x, g^{-1}(t, x)\right)}}=\phi \cdot \partial_{t}\left[v\left(t, x, g^{-1}(t, x)\right)\right]-\phi\left[\left(D_{a} v\right)_{\left.\right|_{\left(t, x, g^{-1}(t, x)\right)}} \partial_{t} g^{-1}(t, x)\right] .
$$

Hence, since $\operatorname{div}_{x}\left[v\left(t, x, g^{-1}(t, x)\right)\right]=\operatorname{div} u(t, x)=0$, it follows using (5.17), (5.18), (5.19) in (5.16) and substituting $v\left(t, x, g^{-1}(t, x)\right)=u(t, x)$ that

$$
\begin{gathered}
\left\langle\partial_{t} m_{\rho_{0}}+\operatorname{div}_{x}\left(v \otimes m_{\rho_{0}}\right), \phi\right\rangle=\int_{[0, T] \times D} \rho(t, x)\left[\partial_{t} u+\operatorname{div} u \otimes u\right] \cdot \phi\left(t, x, g^{-1}(t, x)\right) \\
-\int_{[0, T] \times D} \rho(t, x) \phi\left(D_{a} v\right)_{\left.\right|_{\left(t, x, g^{-1}(t, x)\right)}}\left[\partial_{t} g^{-1}(t, x)+D_{x} g^{-1}(t, x) v\right] d t d x .
\end{gathered}
$$

Next, we write $x=g(t, a)$ for some $a \in D$ and we note that

$$
\begin{gathered}
\partial_{t} g^{-1}(t, g(t, a))+D_{x} g^{-1}(t, g(t, a)) v(t, g(t, a), a) \\
=\partial_{t} g^{-1}(t, g(t, a))+D_{x} g^{-1}(t, g(t, a))\left(\partial_{t} g\right)(t, a)=\partial_{t}\left(g^{-1}(t, g(t, a))\right)=0 .
\end{gathered}
$$

Since this identity holds for any $a \in D$ we find

$$
\partial_{t} g^{-1}(t, x)+D_{x} g^{-1}(t, x) v\left(t, x, g^{-1}(t, x)\right)=0 .
$$

With this observation we conclude that

$$
\begin{aligned}
& \left\langle\partial_{t} m_{\rho_{0}}+\operatorname{div}_{x}\left(v \otimes m_{\rho_{0}}\right), \phi\right\rangle \\
& =\int_{[0, T] \times D} \rho(t, x)\left[\partial_{t} u+\operatorname{div} u \otimes u\right]_{\left.\right|_{(t, x)}} \cdot \phi\left(t, x, g^{-1}(t, x)\right) d t d x .
\end{aligned}
$$

Let us consider the third term in (4.2), involving pressure. Note that the measure $c_{\rho_{0}} / \rho_{0}$ is of the precise form (5.8) so that, according to the observation immediately preceding the statement of this theorem, the product $\left(c_{\rho_{0}} / \rho_{0}\right) \nabla_{x} p$ is a well-defined 
distribution. In [7], Brenier used a complicated extension procedure to give sense to the term $\underline{c} \nabla_{x} p$ as it appears in (4.2), in his context. In the present situation however, because we assume smoothness of $g$ and a specific structure for $c$, we can use a simple microlocal procedure to make sense of $\left(c_{\rho_{0}} / \rho_{0}\right) \nabla_{x} p$. Examining Brenier's construction, one can see that the distribution resulting from Brenier's extension procedure coincides with $\left(c_{\rho_{0}} / \rho_{0}\right) \nabla_{x} p$.

Hence, for the same test function $\phi$ used above, we find, upon changing variables from $a$ to $x$ in (5.9),

$$
\left\langle\frac{c_{\rho_{0}}}{\rho_{0}} \nabla_{x} p, \phi\right\rangle=\int_{[0, T] \times D} \nabla_{x} p(t, x) \cdot \phi\left(t, x, g^{-1}(t, x)\right) d t d x .
$$

Putting together (5.22), (5.23) in (4.2) we conclude that

$$
\int_{[0, T] \times D}\left[\rho(t, x)\left(\partial_{t} u(t, x)+\operatorname{div}_{x}(u \otimes u)(t, x)\right)+\nabla p(t, x)\right] \phi\left(t, x, g^{-1}(t, x)\right) d t d x=0 .
$$

Let us revisit (3.5), integrating against a test function $\varphi \in C_{0}^{\infty}\left(Q^{\prime}\right)$. We find:

$$
\int_{[0, T] \times D} \rho_{0}(a)\left[\partial_{t} \varphi(t, g(t, a), a)+v(t, g(t, a), a) \nabla_{x} \varphi(t, g(t, a), a)\right] d t d a=0 .
$$

Making the change of variables $x=g(t, a)$ and using the fact that

$$
\partial_{t} \varphi\left(t, x, g^{-1}(t, x)\right)=\partial_{t}\left[\varphi\left(t, x, g^{-1}(t, x)\right)\right]-\nabla_{a} \varphi\left(t, x, g^{-1}(t, x)\right) \cdot \partial_{t} g^{-1}(t, x)
$$

and

$$
\nabla_{x} \varphi\left(t, x, g^{-1}(t, x)\right)=\nabla_{x}\left[\varphi\left(t, x, g^{-1}(t, x)\right)\right]-D_{x} g^{-1}(t, x) \nabla_{a} \varphi\left(t, x, g^{-1}(t, x)\right),
$$

we deduce, integrating by parts in time and in space, that

$$
\begin{gathered}
\left.-\int_{[0, T] \times D}\left[\partial_{t} \rho(t, x)+\operatorname{div}(\rho u)\right] \varphi\left(t, x, g^{-1}(t, x)\right)\right] d t d x \\
-\int_{[0, T] \times D} \rho(t, x)\left[\partial_{t} g^{-1}(t, x) \cdot \nabla_{a} \varphi\left(t, x, g^{-1}(t, x)\right)\right. \\
\left.+v\left(t, x, g^{-1}(t, x)\right) D_{x} g^{-1}(t, x) \nabla_{a} \varphi\left(t, x, g^{-1}(t, x)\right)\right] d t d x=0 .
\end{gathered}
$$

We again use (5.21) together with the fact that $u$ is divergence-free to obtain

$$
\int_{[0, T] \times D}\left[\partial_{t} \rho(t, x)+\left(u \cdot \nabla_{x} \rho\right)(t, x)\right] \varphi\left(t, x, g^{-1}(t, x)\right) d t d x=0 .
$$

To complete the proof it is enough to show that, given $f \in C_{0}^{\infty}\left((0, T) \times D^{\circ}\right)$ there exists $\varphi \in C_{0}^{\infty}\left(\stackrel{\circ}{ }^{\prime}\right)$ such that $\varphi\left(t, x, g^{-1}(t, x)\right)=f(t, x)$. Here $\stackrel{\circ}{D}$ and $\dot{Q}^{\prime}$ denote the interior of $D$ and $Q^{\prime}$, respectively.

To see this, fix $f \in C_{0}^{\infty}((0, T) \times \stackrel{\circ}{D})$ and set $K \equiv \operatorname{supp} f \Subset(0, T) \times \stackrel{\circ}{D}$, a compact set. For each $t$ we have $g(t, \cdot) \in G(D)$ so that, in particular, $g(t, \cdot)$ is a diffeomorphism; it follows that $g^{-1}(K) \Subset \stackrel{\circ}{D}$. Thus, there exists an open set $A \Subset \stackrel{D}{ }$ such that $g^{-1}(K) \subset A$.

Let $\theta \in C_{0}^{\infty}(\stackrel{\circ}{D})$ be such that $\theta=1$ on $A$.

Define $\varphi=\varphi(t, x, a)=f(t, x) \theta(a)$. Then,

$$
\varphi\left(t, x, g^{-1}(t, x)\right)=f(t, x) \theta\left(g^{-1}(t, x)\right)=f(t, x) .
$$

This concludes the proof. 
Remark. It is a natural question to ask whether the hypothesis on the regularity of the path $t \mapsto g(t, \cdot)$, which we assumed to be $C^{\infty}$, can be weakened. The reason for requiring that $g$ be $C^{\infty}$ is in order to properly define the restriction of the distribution $\nabla_{x} p(t, \cdot)$ to $Y_{t}$. We note that, once higher regularity of $\nabla_{x} p$ has been established in [1], it may be possible to weaken considerably the regularity requirement on $g$. We will leave this as a topic for future investigation.

Our second consistency result is that any smooth solution of the (classical) Euler equations gives rise to a solution of the relaxed Euler equations. We also show that, for small enough $T$ (satisfying a generalization of Arnold's criterion), this solution is a unique minimizer of the action. These facts were proved by Brenier in the homogeneous case; see Theorem 2.4 in [7. We provide an extension to the variable density case whose proof is a straightforward adaptation of Brenier's arguments.

Theorem 5.3. Let $(u, p, \rho) \in\left(C^{1}([0, T] \times D)\right)^{N} \times\left(C^{1}([0, T] \times D)\right)^{2}$ be a solution of the Euler equations (2.1), with $u$ tangent to $\partial D$. Let $g_{u}=g_{u}(t, x):[0, T] \times D \rightarrow D$ denote the flow induced by $u$ with initial position $x$.

Consider the measures

$$
c_{\rho_{0}}=\rho_{0}(a) \delta_{\left(t, g_{u}(t, a), a\right)} \quad \text { and } \quad m_{\rho_{0}}=\rho_{0}(a) \delta_{\left(t, g_{u}(t, a), a\right)} u(t, x) .
$$

Then, $\left(c_{\rho_{0}}, m_{\rho_{0}}\right)$ is a distributional solution of (3.5), (3.7) and (4.2) in the interior of $Q^{\prime}$.

Moreover, if $T$ is small enough, namely,

$$
\Lambda T^{2}<\pi^{2} \inf _{D} \rho_{0}(a)
$$

where $\Lambda$ is the supremum in $Q$ of the largest eigenvalue of the Hessian matrix of the pressure, then $\left(c_{\rho_{0}}, m_{\rho_{0}}\right)$ given by (5.26) is the unique optimal solution of the relaxed problem associated to the target $h=g_{u}(T, \cdot)$.

The proof is a straightforward adaptation of the argument presented in the proof of Theorem 2.4 of [7. We include details of the final step in the proof of uniqueness, omitted in [7, for the sake of completeness. Assume that $\left(\widetilde{c}_{\rho_{0}}, \widetilde{m}_{\rho_{0}}\right)$ is another optimal solution with $\widetilde{m}_{\rho_{0}}=v \widetilde{c}_{\rho_{0}}$. Easy adaptations of the arguments in [7] yield the following inequality:

$$
\frac{1}{2} \int_{Q^{\prime}}\left|v(t, x, a)-\partial_{t} g(t, a)\right|^{2} d \widetilde{c}_{\rho_{0}}(t, x, a) \leq \frac{1}{2} \int_{Q^{\prime}} \Lambda|x-g(t, a)|^{2} d \frac{\widetilde{c}_{\rho_{0}}(t, x, a)}{\rho_{0}(a)},
$$

for any $\Lambda<\frac{\pi^{2}}{T^{2}} \inf _{D} \rho_{0}(a)$. Let $\Lambda=\theta \inf _{D} \rho_{0}(a)$, for some $\theta<\frac{\pi^{2}}{T^{2}}$. With this notation we obtain that

$$
\frac{1}{2} \int_{Q^{\prime}}\left(\left|v(t, x, a)-\partial_{t} g(t, a)\right|^{2}-\theta|x-g(t, a)|^{2}\right) d \widetilde{c}_{\rho_{0}}(t, x, a) \leq 0 .
$$

As suggested by Brenier we may use, for each fixed $a \in D$, Proposition 3.2 of [7] with $z=z(t)=g(t, a)$ to deduce

$$
\frac{1}{2} \int_{Q^{\prime}}\left(\left|v(t, x, a)-\partial_{t} g(t, a)\right|^{2}-\widetilde{\Lambda}|x-g(t, a)|^{2}\right) d \widetilde{c}_{\rho_{0}}(t, x, a) \geq 0,
$$

for any $\widetilde{\Lambda}<\frac{\pi^{2}}{T^{2}}$. 
From (5.27) and (5.28) we find

$$
\int_{Q^{\prime}}(\widetilde{\Lambda}-\theta)|x-g(t, a)|^{2} d \widetilde{c}_{\rho_{0}}(t, x, a) \leq 0 .
$$

Using $\widetilde{\Lambda}>\theta$ in (5.29) we conclude that

$$
\int_{Q^{\prime}}|x-g(t, a)|^{2} d \widetilde{c}_{\rho_{0}}(t, x, a)=0
$$

and, therefore,

$$
\int_{Q^{\prime}}\left|v(t, x, a)-\partial_{t} g(t, a)\right|^{2} d \widetilde{c}_{\rho_{0}}(t, x, a) \leq 0 .
$$

This implies that the support of $\widetilde{c}_{\rho_{0}}$ is the same as that of $c_{\rho_{0}}$ and that $v(t, x, a) \equiv$ $\partial_{t} g(t, a), \widetilde{c}_{\rho_{0}}$-a.e. $\left(Q^{\prime}\right)$, which concludes the proof of the last step.

We add a couple of comments. First, it is natural to ask whether the improved regularity of the pressure, obtained by Ambrosio and Figalli in [1 for the case of constant density, extends to the nonhomogeneous case. Second, it would be very interesting to obtain numerical methods to approximate optimal solutions of the relaxed problem, in particular to illustrate irregular solutions of the relaxed Euler equations.

\section{ACKNOWLEDGMENTS}

The authors gratefully acknowledge FAPESP Thematic Project \#2007/51490-7.

\section{REFERENCES}

[1] Ambrosio, L. and Figalli, A., On the regularity of the pressure field of Brenier's weak solutions to incompressible Euler equations, Calc. Var. Partial Differential Equations 31 (2008), 497509. MR2372903 (2008m:35272)

[2] Arnold, V. I., Sur la Géométrie Différentielle des Groupes de Lie de Dimension Infinie et ses Applications à L'Hydrodynamique des fluides parfaits, Ann. Inst. Fourier, 16 (1966), 319-361. MR0202082 (34:1956)

[3] Arnold, V. I. and Khesin, B.,Topological Methods in Hydrodynamics, Annual Rev. Fluid Mech. 24 (1992), 145-166. MR1145009 (93d:58020)

[4] Beirão da Veiga, H. and Valli, A., On the Euler equations for nonhomogeneous fluids I, Rend. Sem. Univ. Padova, 63 (1980), 151-168. MR605790 (83d:76029a)

[5] Beirão da Veiga, H. and Valli, A., On the Euler equations for nonhomogeneous fluids II, J. Math. Anal. Appl. 73 (1980), 338-350. MR563987 (83d:76029b)

[6] Brenier, Y., The Least Action Principle and the Related Concept of Generalized Flows for Incompressible Perfect Fluids, J. Amer. Math. Soc. 2 (1989), 225-255. MR.969419 (90g:58012)

[7] Brenier, Y., Minimal Geodesics on Groups of Volume-Preserving Maps and Generalized Solutions of the Euler Equations, Commun. Pure Appl. Math. 52, (1999), 411-452. MR.1658919 (2000c:58020)

[8] Danchin, R. The inviscid limit for density-dependent incompressible fluids Ann. Fac. Sci. Toulouse, ser. 615 (2006), 637-688. MR 2295208 (2008a:35217)

[9] Ebin, D. G. and Marsden J., Groups of Diffeomorphisms and the Motion of an Incompressible Fluid, Ann. of Math. (2) 92 (1970), 102-163. MR0271984 (42:6865)

[10] Hörmander, L., The Analysis of Linear Partial Differential Operators, Vol. I, Springer-Verlag, 1983. MR0717035 (85g:35002a)

[11] Lions, P.-L., Mathematical Topics in Fluid Mechanics, vol. I - Incompressible Models, Oxford Univ. Press, Oxford, 1996. MR 1422251 (98b:76001)

[12] Precioso, J.C., Equações relaxadas para hidrodinâmica ideal, não homogênea, Tese de doutorado, IMECC-UNICAMP, (2005). 
[13] Shnirelman, A. I., The Geometry of the Groups of Diffeomorphisms and the Dynamics of an Ideal Incompressible Fluid, Mat. Sb (N.S.) 128(170), (1985), 82-109, 144. MR805697 (87d:58034)

[14] Shnirelman, A., Attainable diffeomorphisms, Geom. Funct. Anal. 3 (1993), 279-294. MR:1215782 (94f:58021)

[15] Shnirelman, A., Generalized fluid flows, their approximation and applications, Geom. Funct. Anal. 4 (1994), 586-620. MR1296569 (95j:58041)

[16] Villani, C., Topics in Optimal Transportation, Graduate Studies in Math. vol. 58, Amer. Math. Soc., Providence, 2003. MR.1964483 (2004e:90003)

Departamento de Matemática, imecC, Rua Sérgio Buarque de Holanda, 651, Cidade Universitária Zeferino Vaz, Universidade Estadual de Campinas - UNiCAMP, Campinas, SP 13083-859, BRAZIL

E-mail address: mlopes@ime.unicamp.br

Departamento de Matemática, imecC, Rua Sérgio Buarque de Holanda, 651, Cidade Universitária Zeferino Vaz, Universidade Estadual de CAMPinas - UNICAMP, CAmpinas SP 13083-859, BRAZIL

E-mail address: hlopes@ime.unicamp.br

Departamento de Matemática, Universidade Estadual Paulista - UnesP, 15054-000, S. J. do Rio Preto, SP, Brazil

E-mail address: precioso@ibilce.unesp.br 